\title{
Minimum Breadth of a Graph
}

\author{
Tabitha Agnes $\mathrm{M}^{*}$ and L Sudershan Reddy ${ }^{\dagger}$
}

\begin{abstract}
Breadth of a graph as the maximum of heights taken over all diametral paths is investigated in [3, 4], where height is taken by placing each diametral path on level $y=0$ and placing uniquely the rest of the vertices on levels $y=1$, $2 \ldots \mathrm{k}$ keeping adjacency intact. A parameter minimum breadth is introduced as minimum of heights with respect to all diametral paths. A few results on minimum breadth in certain classes of graphs are presented. Also the bounds on number of vertices and edges for graphs of known diameter and minimum breadth are proposed.
\end{abstract}

Keywords: Diameter, Radius, Peripheral vertices, Central vertices, Diametral Path, Breadth.

Mathematics Subject Classification (2010): $05 C 12$

\section{Introduction}

The concept of a diametral path in graphs is of great interest to researchers due to its importance in the study of networks. The concepts related to diameter, peripheral vertices and diametral paths have been investigated in literature [5]. The identification of the diametral path to which all the other vertices are at closest distance possible offers solution to many problems in transportation, distribution, designing, communication, team formation and event management.

\footnotetext{
* Research Scholar, Visvesvaraya Technological University, Belgaum, India; tabithaagnesm@yahoo.co.in

† Professor, C M S Business School, Jain University, Bangalore, India; sudershan.reddy@cms.ac.in
} 
In this paper, a study is undertaken on diametral paths and the parameter minimum breadth. In Section 2, the parameter minimum breadth is introduced and few results on minimum breadth in certain classes of graphs are presented. In Section 3, bounds on number of vertices and edges are determined for graphs of known diameter.

The length of a path is the number of edges on the path. The distance between two vertices in a graph is the length of shortest path between them. The eccentricity of a vertex is the maximum of distances from it to all the other vertices of that graph. While diameter is the maximum of the eccentricities of all vertices of that graph, the radius is minimum. Peripheral vertices are vertices of maximum eccentricity and central vertices are of minimum eccentricity. The diametral path of a graph is the shortest path between two vertices which has length equal to diameter of that graph.

The graphs considered in this paper, are simple, connected, undirected, labelled and unweighted graphs. The definitions and results are in accordance with [1,2].

Given below are a few standard results on diameter in certain classes of graphs.

1. Complete graph $K_{n}: \operatorname{diam}\left(K_{n}\right)=1$ where $n \geq 2$.

2. Wheel $W_{n}$ : $\operatorname{diam}\left(W_{n}\right)=2$ where $n \geq 5$.

3. Star $K_{1, n}: \operatorname{diam}\left(K_{1, n}\right)=2$ where $n \geq 2$.

4. Complete bipartite graph $K_{m, n}: \operatorname{diam}\left(K_{m, n}\right)=2$ where $m \geq 2$ or $\mathrm{n} \geq 2$.

5. Path $P_{n}: \operatorname{diam}\left(P_{n}\right)=n-1$ and $\operatorname{rad}\left(P_{n}\right)=\lfloor n / 2\rfloor$.

6. Cycle $C_{n}: \operatorname{diam}\left(C_{n}\right)=\lfloor n / 2\rfloor$.

\section{Minimum Breadth of a Graph}

The graph is redrawn by considering the first quadrant of $X Y$ plane with auxiliary lines $y=0,1,2 \ldots k$. The diametral path is placed on line $y=0$ and vertices that are adjacent to vertices of diametral path are placed on the line $y=1$. The vertices that are adjacent to the 
vertices of the line $y=1$ (excluding vertices on level $y=0$ ) are placed on the line $y=2$. The procedure is continued till all the vertices of the graph are placed uniquely.

Definition 2.1 If there exists at least one vertex on the line $y=h$ and no vertices on the line $y=h+1$, then the height of the graph with respect to that diametral path is taken to be $h$.

Definition 2.2 Minimum breadth $\left(\mathrm{Br}_{\mathrm{m}}\right)$ of a graph is defined to be the minimum of the heights with respect to all the diametral paths.

$\operatorname{Br}_{m}(G)=\operatorname{Min}\left\{h_{i}\right\}$ where $h_{i}$ is the height with respect to $i^{\text {th }}$ diametral path.

Example 2.1 Consider the following graph in figure 1(a). Here $\operatorname{diam}(G)=3$. Diametral paths are ABFE, AGFE and ABDE. It can be noted that $h_{1}=1, h_{2}=2$ and $h_{3}=1$ from figures $1(b), 1(c)$ and $1(d)$. $\operatorname{Br}_{\mathrm{m}}(\mathrm{G})=\operatorname{Min}\{1,2,1\}=1$.

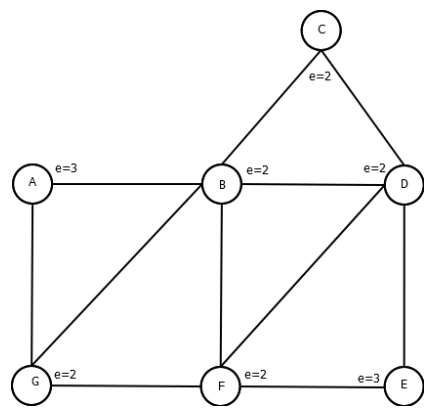

Fig 1(a)

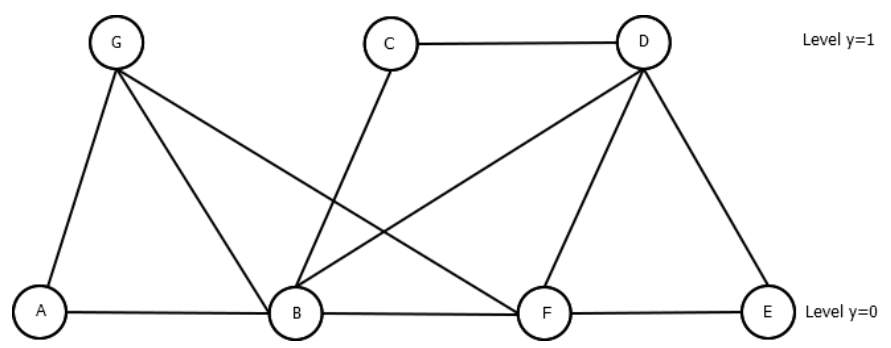



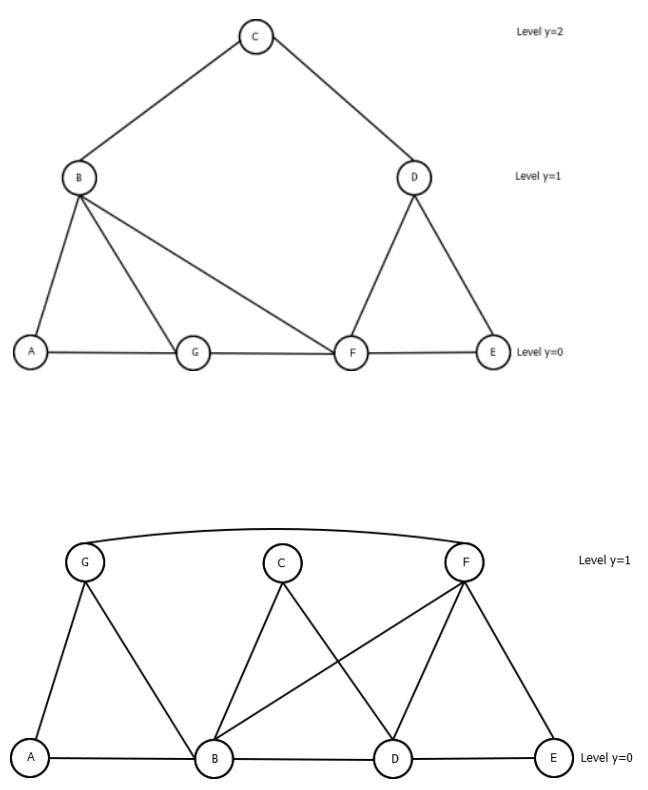

Fig 1(b, c, d)

Proposition 2.1 For a path $\mathrm{P}_{\mathrm{n}}, \mathrm{Br}_{\mathrm{m}}\left(\mathrm{P}_{\mathrm{n}}\right)=0$.

Proof: Since diameter of path is equal to length of the path, diametral path is the path itself. Hence there will be no vertices on the line $\mathrm{y}=1$ and $\operatorname{Br}\left(\mathrm{P}_{\mathrm{n}}\right)=0$.

Proposition 2.2 For a complete graph $K_{n}$ where $n \geq 2, B_{m}\left(K_{n}\right)=1$.

Proof: Since $\operatorname{diam}\left(K_{n}\right)=1$, every edge is a diametral path. Placing any edge on level $y=0$, the remaining vertices will be on level $y=1$ as they are adjacent to both the vertices on level $y=0$.

Hence $\mathrm{Br}_{\mathrm{m}}\left(\mathrm{K}_{\mathrm{n}}\right)=1$.

Proposition 2.3

For a complete bipartite graph $K_{m, n}(m \geq 2$ or $n \geq 2), B_{m}\left(K_{m, n}\right)=1$.

Proof: Since $\operatorname{diam}\left(\mathrm{K}_{\mathrm{m}, \mathrm{n}}\right)=2$, every diametral path has two vertices of a partite and one vertex of the other partite of the graph. Placing a diametral path on level $y=0$, all the other vertices are on line $y=$ 1 as they are adjacent to atleast one of the vertices of the diametral path. 
Example 2.2 Consider a complete bipartite graph $\mathrm{K}_{3,2}$ in figure 2(a). $\operatorname{diam}\left(\mathrm{K}_{3,2}\right)=2$. Placing diametral path ADC on level $\mathrm{y}=0$, we get $\mathrm{Br}_{\mathrm{m}}\left(\mathrm{K}_{3,2}\right)=1$ as noted in figure 2(b).
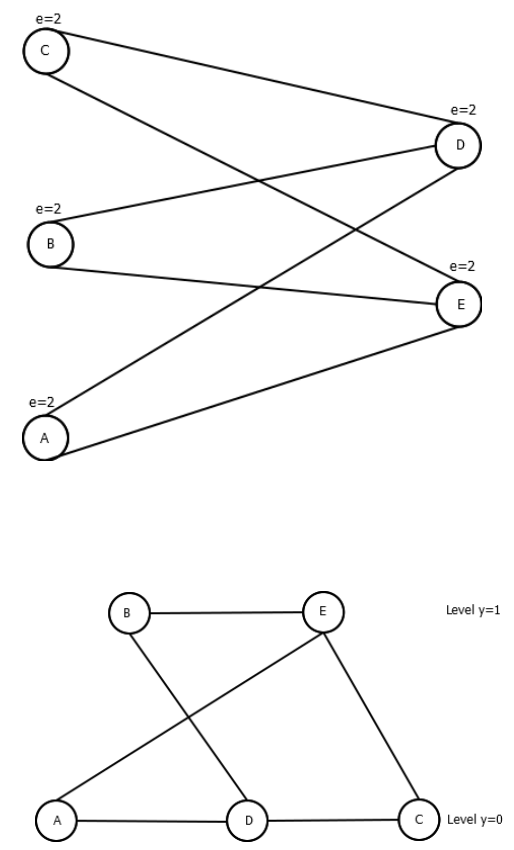

Fig.2 (a). Complete bipartite graph $\mathbf{K}_{\mathbf{m}, \mathbf{n}}$ Fig.2(b).

Proposition 2.4 For cycles $C_{n}$,

[a] $\mathrm{Br}_{\mathrm{m}}\left(\mathrm{C}_{\mathrm{n}}\right)=\lfloor(\mathrm{n}-\mathrm{d}) / 2\rfloor$

[b] $\mathrm{Br}_{\mathrm{m}}\left(\mathrm{C}_{\mathrm{n}}\right)=\mathrm{k}$ where $\mathrm{k} \geq 1$ and $\mathrm{n}=4 \mathrm{k}$ or $\mathrm{n}=4 \mathrm{k}-1$ or $\mathrm{n}=4 \mathrm{k}+1$ or $\mathrm{n}$ $=4 \mathrm{k}+2$.

\section{Proof:}

[a] $\operatorname{diam}\left(\mathrm{C}_{\mathrm{n}}\right)=\mathrm{d}=[\mathrm{n} / 2\rfloor$. Placing diametral path with $\mathrm{d}+1$ vertices on level $\mathrm{y}=0$, the remaining $\mathrm{n}-\mathrm{d}-1$ vertices are arranged in $\Gamma(\mathrm{n}-$ $\mathrm{d}-1) / 2\rceil=\lfloor(\mathrm{n}-\mathrm{d}) / 2\rfloor$ levels with a maximum of two vertices on each level.

Hence $\operatorname{Br}_{m}\left(C_{n}\right)=\lfloor(n-d) / 2\rfloor$.

[b] When $n=4 k$ or $n=4 k-1$ or $n=4 k+1$ or $n=4 k+2$, 
then $\mathrm{d}=2 \mathrm{k}$ or $\mathrm{d}=2 \mathrm{k}-1$ or $\mathrm{d}=2 \mathrm{k}$ or $\mathrm{d}=2 \mathrm{k}+1$ respectively. Then the remaining number of edges are $2 \mathrm{k}$ or $2 \mathrm{k}$ or $2 \mathrm{k}+1$ or $2 \mathrm{k}+1$ respectively. Placing $d$ edges on diametral path, the remaining edges are placed in such a way that there are minimum two edges incident on each level beginning from level $y=1$. The level number at which the last two edges are incident is the minimum breadth of the graph. Hence the minimum breadth of the graph is $k$ respectively in all the cases. Hence $\mathrm{Br}_{\mathrm{m}}\left(\mathrm{C}_{\mathrm{n}}\right)=\mathrm{k}$.

\section{Example 2.3}

Consider a cycle $\mathrm{C}_{6}$ in figure $3(\mathrm{a}) \cdot \operatorname{diam}\left(\mathrm{C}_{6}\right)=3$. Placing diametral path $A B C D$ on level $y=0$, we get $\operatorname{Br}\left(C_{6}\right)=1$ as noted in figure $3(b)$.
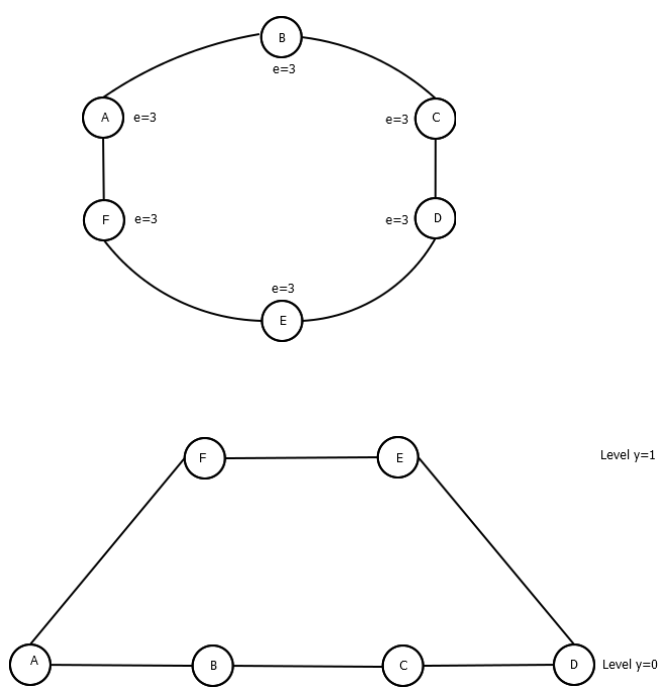

Fig.3(a).Cycle $\mathrm{C}_{6}$ Fig.3(b).

Proposition 2.5 For a wheel $\mathrm{W}_{\mathrm{n}}(\mathrm{n} \geq 5), \quad \mathrm{Br}_{\mathrm{m}}\left(\mathrm{W}_{\mathrm{n}}\right)=1$.

Proof: $\operatorname{diam}\left(\mathrm{W}_{\mathrm{n}}\right)=2$. Consider a diametral path that passes through a central and has two non adjacent peripheral vertices as end vertices. By placing this diametral path on level $y=0$, the remaining vertices are all adjacent to central vertex and lie on level $\mathrm{y}=1$. Hence $\mathrm{Br}_{\mathrm{m}}\left(\mathrm{W}_{\mathrm{n}}\right)=1$. 


\section{Example 2.4}

Consider a wheel $\mathrm{W}_{5}$ in figure $4(\mathrm{a})$.

$\operatorname{diam}\left(\mathrm{W}_{\mathrm{n}}\right)=2$. Placing diametral path AEC on level $\mathrm{y}=0$, we get $\mathrm{Br}_{\mathrm{m}}\left(\mathrm{W}_{\mathrm{n}}\right)=1$ as noted in figure $4(\mathrm{~b})$.
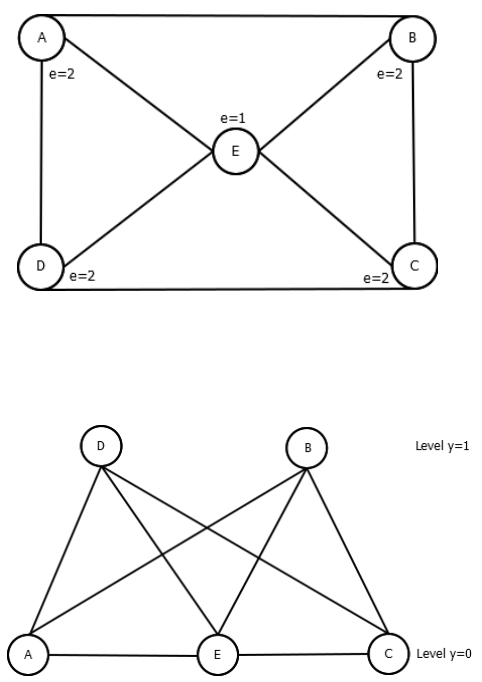

Fig.4(a) Wheel $W_{n}$ Fig.4(b).

Proposition 2.6 For a star $\mathrm{K}_{1, \mathrm{n}}(\mathrm{n} \geq 2), \mathrm{Br}_{\mathrm{m}}\left(\mathrm{K}_{1, \mathrm{n}}\right)=1$.

Proof: Since $\operatorname{diam}\left(\mathrm{K}_{1, \mathrm{n}}\right)=2$, the diametral path has a central and two peripheral vertices on level $y=0$. The remaining vertices are all adjacent to central vertex and lie on level $y=1$.

Hence $\operatorname{Br}\left(\mathrm{K}_{1, \mathrm{n}}\right)=1$.

Example 2.5

Consider a star $\mathrm{K}_{1,4}$ in figure $5(\mathrm{a}) \cdot \operatorname{diam}\left(\mathrm{K}_{1,4}\right)=2$. Placing the diametral path AEC on level, we get $\mathrm{Br}_{\mathrm{m}}\left(\mathrm{K}_{1,4}\right)=1$ as noted in figure $5(b)$. 

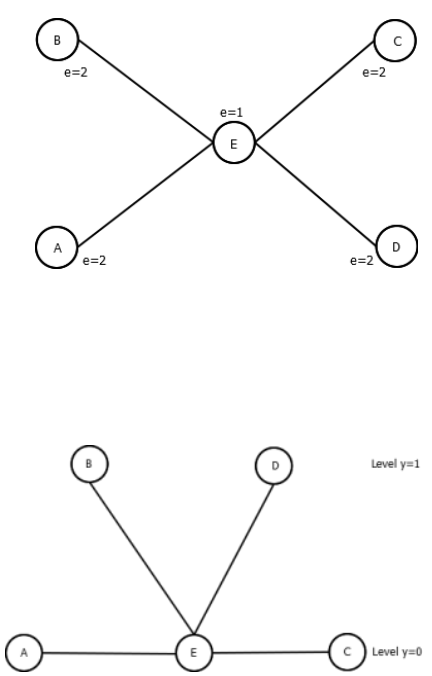

Fig.5(a).Star $\mathrm{K}_{1,4}$ Fig.5(b).

\section{Bounds}

Theorem 3.1 If $\mathrm{Br}_{\mathrm{m}}(\mathrm{G})=\mathrm{b}$, $\operatorname{diam}(\mathrm{G})=\mathrm{d}$ and $\mathrm{d} \geq 2 \mathrm{~b}$,

then $\mathbf{n} \geq \mathbf{d}+\mathbf{b}+\mathbf{1}$ and $\mathbf{m} \geq \mathbf{d}+\mathbf{b}$ where $\mathrm{m}, \mathrm{n}$ are the number of edges and vertices respectively in a graph $\mathrm{G}$.

Proof: Since $\operatorname{diam}(G)=d$, there are $d+1$ vertices on level $y=0$.

Since $\mathrm{Br}_{\mathrm{m}}(\mathrm{G})=\mathrm{b}$,

there is at least one vertex each on levels from $y=1$ to $y=b$. Hence there are at least $b$ vertices on levels $y=1$ to $y=b$.

The minimum number of vertices $=d+1+b$. Hence $\mathbf{n} \geq \mathbf{d}+\mathbf{b}+\mathbf{1}$.

Since $\operatorname{diam}(G)=d$, there are d edges on level $y=0$.

Since $\operatorname{Br}_{\mathrm{m}}(G)=b$, there is at least one vertex each on levels from $\mathrm{y}=$ 1 to $y=b$. Since there is at least one edge between these levels $y=0$ and $y=1$, the minimum number of edges is $d+b$. Also the vertex on level $y=1$ is adjacent to one or more of the vertices which are at a distance greater than or equal to $b$ from both peripheral vertices on level $y=0$.(Otherwise diameter increases.) 
Hence, $\mathbf{m} \geq \mathbf{d}+\mathbf{b}$.

We can conclude that $\mathbf{n} \geq \mathbf{d}+\mathbf{b}+\mathbf{1}$ and $\mathbf{m} \geq \mathbf{d}+\mathbf{b}$.

Example 2.6 Consider graphs for which $\mathrm{Br}_{\mathrm{m}}(\mathrm{G})=\mathrm{b}=2$.

Since $\operatorname{diam}(G)=d$ and $d \geq 2 b$, we take cases $d=4$ and $d=5$.

When $b=2$ and $d=4$, we get $n=7$ and $m=6$ in figure $6(a)$.

When $b=2$ and $d=5$, we get $n=8$ and $m=7$ in figure $6(b)$.

It can be noted that $n \geq d+b+1$ and $m \geq d+b$ in each case.
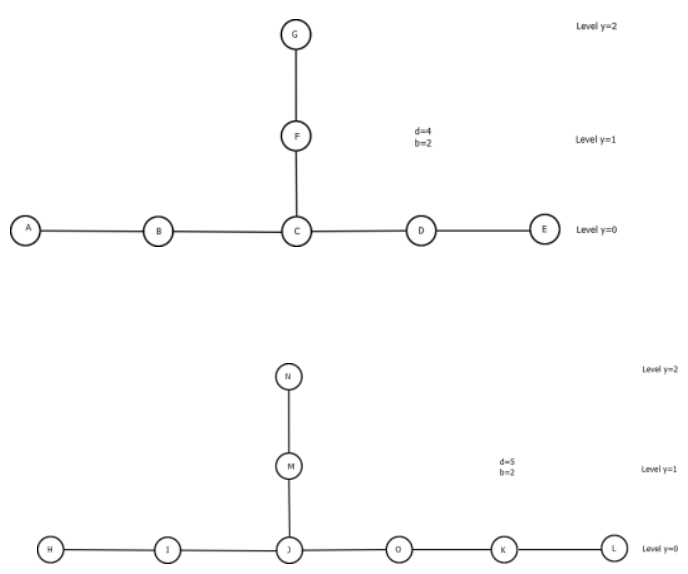

Fig 6(a) Fig 6(b)

Theorem 3.2 If $\operatorname{Br}(G)=b$, $\operatorname{diam}(G)=d$ and $d=2 b-1$ or $d=2 b-2$, then $\mathbf{m} \geq \mathrm{d}+\mathrm{b}+\mathbf{1}$ and $\mathbf{n} \geq \mathrm{d}+\mathrm{b}+\mathbf{1}$.

\section{Proof:}

Case 1: The expressions $d=2 b-1$ and $d+1=2(d-b)+2$ are equivalent.

Since $d=2 b-1$, the number of edges on level $y=0$ is $d$ and the number of vertices on level $\mathrm{y}=0$ is $\mathrm{d}+1=2(\mathrm{~d}-\mathrm{b})+2$.

As the diameter increases, the vertex on level $y=1$ is adjacent to only 2 vertices excluding $(d-b)$ vertices from each end vertex on 
the diametral path. So there are at least 2 edges between levels $y=0$ and $\mathrm{y}=1$.

Case 2: The expressions $d=2 b-2$ and $d+1=2(d-b)+3$ are equivalent.

Since $d=2 b-2$, the number of edges on level $y=0$ is $d$ and the number of vertices on level $\mathrm{y}=0$ is $\mathrm{d}+1=2(\mathrm{~d}-\mathrm{b})+3$.

As the diameter increases, the vertex on level $y=1$ is adjacent to at least 2 of the 3 vertices excluding $(d-b)$ vertices from each end vertex on the diametral path. So there are at least 2 edges between levels $\mathrm{y}=0$ and $\mathrm{y}=1$.

Hence we can conclude that the minimum number of edges

$=\mathrm{d}($ on level $\mathrm{y}=0)+2($ between levels $\mathrm{y}=0$ and $\mathrm{y}=1)+\mathrm{b}-$ 1 (between levels $\mathrm{y}=1$ and $\mathrm{y}=\mathrm{b}$ )

$=\mathrm{d}+\mathrm{b}+1$.

Also the minimum number of vertices $=\mathrm{d}+1($ on level $\mathrm{y}=0)+\mathrm{b}$ (one vertex each on levels $y=1$ to $y=b)=d+b+1$.

Hence $\mathbf{m} \geq \mathbf{d}+\mathbf{b}+\mathbf{1}$ and $\mathbf{n} \geq \mathbf{d}+\mathbf{b}+\mathbf{1}$.

Example 2.7 Consider graphs for which $\mathrm{Br}_{\mathrm{m}}(\mathrm{G})=\mathrm{b}=4$.

Since $\operatorname{Diam}(G)=d$ and $d=2 b-1$ or $d=2 b-2$, we take cases $d=7$ and $\mathrm{d}=6$.

When $b=4$ and $d=7$, we get $n=12$ and $m=12$ in figure $7(a)$.

When $b=4$ and $d=6$, we get $n=11$ and $m=11$ in figure $7(b)$.

It can be noted that $\mathbf{n} \geq \mathbf{d}+\mathbf{b}+\mathbf{1}$ and $\mathbf{m} \geq \mathbf{d}+\mathbf{b}+\mathbf{1}$ in each case.

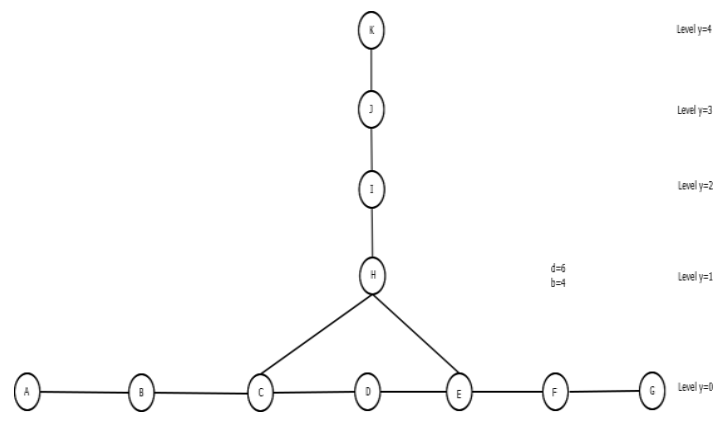




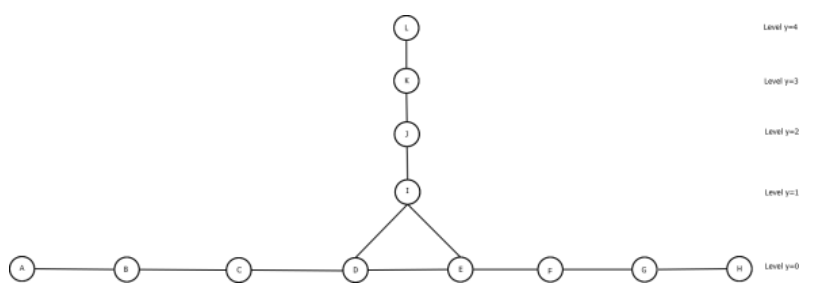

Fig 7(a) Fig 7(b)

\section{Conclusion}

In this paper, a few results and bounds related to the introduced parameter minimum breadth are presented. Since diameter is the maximum of all distances in a graph, the parameter minimum breadth is less than diameter. The smaller the value of $\mathrm{Br}_{\mathrm{m}}$, the closer the vertices are to a particular diametral path. The bounds of number of vertices and edges in graphs with $d \geq 2 b-2$ are discussed. Further to this study, the focus would be on bounds for $\mathrm{d} \leq 2 \mathrm{~b}-3$ and the applications of this parameter. It remains a challenge to determine how a graph can be restructured with addition or deletion of vertices or edges to attain a smaller value of $\mathrm{Br}_{\mathrm{m}}$.

\section{References}

[1] F. Buckley and F. Harary, Distance in Graphs, Perseus Books, 1990.

[2] F. Harary, Graph Theory, Addison-Wesley, 1969.

[3] N. Kishori, Some aspects of distance concepts in graph theory. Ph. D, Thesis. Karnatak University, Dharwad, pp.81-101, 2007.

[4] H. B. Walikar, S. V. Shinde, I. Baidri and R. H. Roogi, "Square Graphs" an Algorithmic Approach, volume 3, no. 4, International Journal of Advanced Research in Computer Science, 2012.

[5] J. S. Deogun and D. Kratsch, "Diametral Path Graphs," Graph-Theoretic Concepts in Computer Science, volume 1017, 1995, pp 344-357. 